\title{
Intensity limitations due to space charge for bunch compression in synchrotrons
}

\author{
Yao-Shuo Yuan, ${ }^{1}$ Oliver Boine-Frankenheim, ${ }^{1,2}$ and Ingo Hofmann ${ }^{1,2}$ \\ ${ }^{1}$ Technische Universität Darmstadt, Schlossgartenstrasse 8, 64289 Darmstadt, Germany \\ ${ }^{2}$ GSI Helmholtzzentrum für Schwerionenforschung GmbH, Planckstrasse 1, 64291 Darmstadt, Germany
}

(Received 20 March 2018; published 9 July 2018)

\begin{abstract}
Bunch compression achieved via a fast bunch rotation in longitudinal phase space is a well-accepted scheme to generate short, intense ion bunches for various applications. During bunch compression, coherent beam instabilities and incoherent single particle resonances can occur because of increasing space charge, resulting in an important limitation for the bunch intensity. We present an analysis of the relevant space charge driven beam instability and resonance phenomena during bunch compression. A coupled longitudinal-transverse envelope approach is compared with particle-in-cell (PIC) simulations. Two distinct cases of crossing are discussed and applied to the GSI SIS-18 heavy-ion synchrotron. It is shown that during bunch compression, the $90^{\circ}$ condition of phase advance is associated with a fourth order single particle resonance and the $120^{\circ}$ condition with the recently discovered dispersion-induced instability. The agreement between the envelope and PIC results indicates that the stop band is defined by the $120^{\circ}$ dispersion instability, which should be avoided during bunch compression.
\end{abstract}

DOI: 10.1103/PhysRevAccelBeams.21.074201

\section{INTRODUCTION}

Short and intense ion or proton bunches are required for many applications, such as the production and subsequent storage of exotic nuclei or antiprotons [1], generation of dense plasmas and spallation neutron sources. Short bunches can be obtained by longitudinal rf bunch compression just before extraction from a synchrotron. Space charge represents an important limitation for the maximum compression ratio as the increasing transverse space charge tune spread leads to resonance crossing and corresponding emittance growth. Therefore, the bunch compression process must be completed as fast as possible in order to minimize the dwelling time of bunches in the extreme space charge regime.

A widely-accepted scheme to achieve bunch compression is a $90^{\circ}$ nonadiabatic fast bunch rotation in mismatched $\mathrm{rf}$ bucket. For instance, at GSI, such a fast bunch rotation process is performed before extraction in the SIS-18 [2,3], and proposed for the FAIR SIS-100 [4].

The scheme of bunch compression based on fast rotation has been the subject of numerous theoretical studies investigating instability limits and beam quality evolution [5-11], based on the envelope equation approach [12] as

Published by the American Physical Society under the terms of the Creative Commons Attribution 4.0 International license. Further distribution of this work must maintain attribution to the author(s) and the published article's title, journal citation, and DOI. well as particle-in-cell (PIC) simulations. Studies on bunch compression with space charge near transition energy have been carried out (see, for example, [13]), also bunch rotation schemes for rapid cycling synchrotrons [14].

The bunch compression in SIS-18 is usually performed with phase advances per periodic cell in horizontal and vertical directions larger than $120^{\circ}$, respectively $90^{\circ}$. In the vertical plane, and for very high intensity, the space-charge depressed phase advance per cell crosses dynamically $90^{\circ}$. Thus, a fourth-order single particle resonance and the wellknown envelope instability - both entirely driven by space charge-are of potential concern with practically overlapping stop bands [15]. Furthermore, in the horizontal plane, bunch compression may lead to crossing of $120^{\circ}$, and the recently discovered dispersion-induced instability [16] could be a potential limitation of bunch intensity.

The present study investigates bunch dynamics during compression for parameters of the SIS-18. The lattice is assumed to be linear and the nonlinearities result from the space charge force only. Based on the coupled longitudinaltransverse envelope equations and PIC simulations, the intensity limitation during bunch compression related to the coherent beam instability (envelope instability and dispersion-induced instability) and incoherent single particle resonance (fourth-order resonance) are analyzed in detail.

The article is structured as follows. At first, we introduce the theoretical model of the coupled longitudinal-transverse envelope equation set and PIC simulations of bunch compression. Second, as an application of the two approaches to describe the bunch compression in SIS-18, we solve 
numerically the coupled envelope equations for typical SIS-18 beam parameters, in comparison with PIC simulation results. At last, two major space-charge limits for bunch compression in SIS-18 with phase advance crossing the resonance conditions related to $90^{\circ}$ and $120^{\circ}$ line are investigated. The competing mechanisms of the fourth-order resonance, envelope instability and dispersion-induced instability during bunch compression are demonstrated.

\section{APPROACHES TO BUNCH COMPRESSION STUDIES}

The envelope approach and PIC simulations are two commonly used methods in bunch compression studies. Based on the longitudinal envelope equation, the required rf voltage for bunch rotation can be calculated [13]. The transverse space-charge tune shift during bunch compression can be obtained by solving the transverse envelope equation set [11]. This chapter introduces the coupled longitudinal-transverse envelope equation set, and briefly discusses several considerations for performing simulations of bunch compression. In the following we assume $x$ and $y$ for the horizontal and vertical directions, and $z$ the longitudinal one; $s$ is distance as independent variable. $k_{0, x, y}, k_{x, y}$ are the phase advances per focusing cell without and with space charge, respectively.

\section{A. Longitudinal beam dynamics}

Let us start with a bunch with parabolic distribution in the longitudinal plane, for which the space charge forces are linear in $z$. The corresponding longitudinal envelope equation including space charge takes the form [17]

$$
z_{m}^{\prime \prime}+\kappa_{z 0} z_{m}-\frac{K_{L}}{z_{m}^{2}}-\frac{\varepsilon_{L}^{2}}{z_{m}^{3}}=0 .
$$

Here, $z_{m}$ is the half length of the bunch, and $\kappa_{z 0}$ represents the linearized external rf focusing force constant given by

$$
\kappa_{z 0}=q Z V_{r f} h \eta /\left(2 \pi R^{2} \gamma \beta^{2} A m_{0} c^{2}\right) .
$$

$V_{r f}$ is the rf voltage required for fast bunch compression, $Z q$ and $A m_{0}$ the charge and the mass of the particle, $h$ the harmonic number, $\eta$ the slip factor, $c$ the speed of light, $\beta$ and $\gamma$ the relativistic factors, and $R$ the average radius of the synchrotron. The linearized external focusing gradient modified by longitudinal space charge is $\kappa_{z}=\kappa_{z 0}-K_{L} / z_{m}^{3}$, with the longitudinal perveance $K_{L}=3 g N Z^{2} r_{p} /\left(2 \beta^{2} \gamma^{5} A\right)$; the geometry factor $g=0.5+2 \ln \left(R_{p} / R_{b}\right)\left(R_{p}\right.$ and $R_{b}$ are the radii of the beam pipe and the beam, respectively); $N$ the number of particles in the bunch; $r_{p}$ the classical proton radius and $\eta$ the slip factor. $\varepsilon_{L}$ is the longitudinal emittance, defined as

$$
\varepsilon_{L}=|\eta| z_{m} \delta_{0}
$$

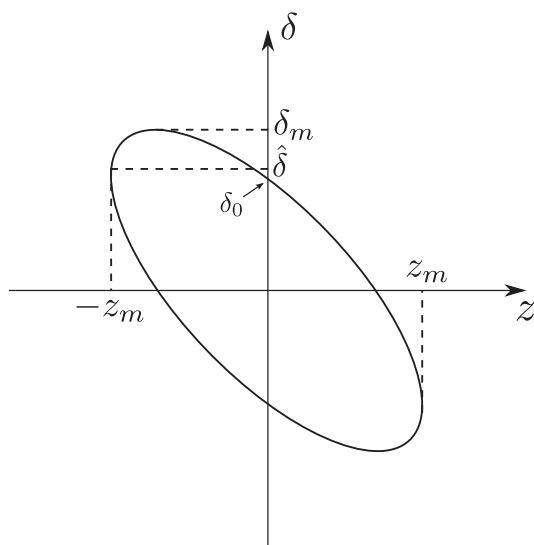

FIG. 1. Definition of parameters in longitudinal phase space. $z$ denotes the longitudinal direction, and $\delta=\Delta p / p_{0}$ is the relative momentum deviation.

in which $\delta_{0}$ is the momentum spread at the center of the bunch $\delta_{0}=\delta(z=0)$, as shown in Fig. 1. Physically, $\varepsilon_{L}$ denotes the area in longitudinal phase space, which is constant without acceleration. During compression, the ellipse of the longitudinal emittance rotates and becomes tilted, and the maximum momentum spread of the bunch $\tilde{\delta}$ can be expressed as $\delta_{m}^{2}=\hat{\delta}^{2}+\delta_{0}^{2}$. Here, $\hat{\delta}$ is the momentum spread of the particles at the edge of the bunch, and $\delta_{0}$ the maximum momentum spread at the bunch center.

The rf voltage required for bunch compression can be derived by integrating Eq. (1) [13],

$\int \mathrm{d}\left(\frac{z_{m}^{\prime 2}}{2}\right)+\kappa_{z 0} \int z_{m} \mathrm{~d} z_{m}-K_{L} \int \frac{\mathrm{d} z_{m}}{z_{m}^{2}}-\varepsilon_{L}^{2} \int \frac{\mathrm{d} z_{m}}{z_{m}^{3}}=0$

from which we obtain a longitudinal invariant,

$$
I_{L}=\frac{z_{m}^{\prime 2}}{2}+\frac{\kappa_{z 0}}{2} z_{m}^{2}+\frac{K_{L}}{z_{m}}+\frac{\varepsilon_{L}^{2}}{2 z_{m}^{2}} .
$$

It is convenient to introduce a compression factor $\chi(\chi \leq 1)$, defined as

$$
z_{m, f}=\chi z_{m, i},
$$

in which $z_{m, f}$ and $z_{m, i}$ denote the initial and final longitudinal envelope during bunch compression. For the ideal bunch compression with a parabolic distribution and linear longitudinal space charge force, the longitudinal emittance $\varepsilon_{L}$ remains invariant, and the final momentum spread results as $\delta_{m, f}$ obeys $\delta_{m, f}=\frac{\delta_{m, i}}{\chi}$.

Substituting $z_{m, i}, z_{m, f}$ and $\delta_{m, i}, \delta_{m, f}$ into the invariant emittance of Eq. (4), and using the compression factor $\chi$, we can obtain

$$
\kappa_{z 0}=\left(\frac{|\eta| \delta_{m, i}}{z_{m, i} \chi}\right)^{2}\left[1+\frac{2 K_{L} z_{m, f}}{\varepsilon_{L}^{2}(1+\chi)}\right] .
$$


The required rf voltage for bunch compression can be derived via substituting Eq. (7) into Eq. (2),

$V_{r f}=\left(\frac{|\eta| \delta_{m, i}}{z_{m, i} \chi}\right)^{2}\left[1+\frac{2 K_{L} z_{m, i} \chi}{\varepsilon_{L}^{2}(1+\chi)}\right] \frac{2 \pi R^{2} \gamma \beta^{2} A m_{0} c^{2}}{Z q h \eta}$.

It can be seen from Eq. (8) that the rf voltage for the bunch compression is a function of the initial bunch length $z_{m, i}$, momentum spread $\delta_{m, i}$ and the compression factor $\chi . V_{r f}$ is inversely proportional to the bunch compression factor $\chi$, or the final bunch length $z_{m, f}$.

Since during bunch compression the synchrotron phase are chosen to be zero, the synchrotron frequency can be written as (see, for example, in Ref. [18])

$$
\omega_{s}=\sqrt{\frac{Z q h V_{r f}|\eta|}{2 \pi R^{2} A m_{0} \gamma}} .
$$

Since bunch compression are performed by one quarter synchrotron motion, the turns needed for compression are

$$
n=\frac{1}{4} \frac{\omega_{0}}{\omega_{s}},
$$

where $\omega_{0}=\beta_{0} c / R$ is the revolution frequency. Note that in Eqs. (9) and (10) the longitudinal space charge is ignored. For the parameters in the present study, longitudinal space charge is sufficiently small that Eq. (10) can be used to calculate the turns required for bunch compression.

\section{B. Coupled longitudinal-transverse envelope system}

The longitudinal envelope equation of Eq. (1) can be used to describe the longitudinal beam dynamics and calculate the required rf voltage in bunch compression. By including the space-charge modified dispersion, the transverse envelope equations become a useful tool to study the transverse collective motion in synchrotrons (for example, see Refs. [16,19]). During bunch compression, both space charge and its effect on dispersion are increasing. Therefore, the transverse envelope equations for a given slice of a long bunch-or just the bunch center as assumed here-should be coupled with the longitudinal equation to give a full description of the beam dynamics of bunch compression.

There are several considerations when applying the coupled set of equations on bunch compression. The first consideration is that the coupled envelope equations discussed here by using Eq. (1) apply to bunch lengths by far larger than the transverse sizes of the beam pipe. This is different from the case of a high current linac, where a symmetrical three-dimensional set of envelope equations is often used (for example, see Refs. [12,20]). Second, even for fast bunch rotation, the synchrotron frequency is much smaller than the transverse one, which means space charge is increasing "adiabatically" for the transverse motion. Therefore, it is reasonable to assume that the space charge perveance in transverse envelope equations remains a constant for each turn, and the beam remains matched to the transverse lattice during bunch compression. For progressive turns during bunch compression the transverse space charge perveance is changing as it depends on the number of particles per unit length of the considered slice. The third point is that the variable $z_{m}$ in the longitudinal equation should be transformed to its corresponding rms quantity $\sigma_{z}$, in order to calculate the rms momentum spread and dispersion. For parabolic distributions the transformation is performed by $\sigma_{z}=\sqrt{5} z_{m}$. Based on above considerations, the coupled longitudinal-transverse set of equations can be written in the following form by using the generalized transverse rms emittances introduced in Ref. [21]:

$$
\begin{aligned}
& z_{m}^{\prime \prime}+\left(\kappa_{z 0}-\frac{K_{L}}{z_{m}^{3}}\right) z_{m}-\frac{\varepsilon_{z}^{2}}{z_{m}^{3}}=0, \\
& \sigma_{x}^{\prime \prime}+\left[\kappa_{x 0}-\frac{K_{\mathrm{sc}}}{2 X(X+Y)}\right] \sigma_{x}-\frac{\varepsilon_{d x}^{2}}{\sigma_{x}^{3}}=0, \\
& \sigma_{y}^{\prime \prime}+\left[\kappa_{y 0}-\frac{K_{\mathrm{sc}}}{2 Y(X+Y)}\right] \sigma_{y}-\frac{\varepsilon_{d y}^{2}}{\sigma_{y}^{3}}=0, \\
& D_{x}^{\prime \prime}+\left[\kappa_{x 0}-\frac{K_{\mathrm{sc}}}{2 X(X+Y)}\right] D_{x}=\frac{1}{\rho(s)},
\end{aligned}
$$

with the coupled relations

$$
X=\sqrt{\sigma_{x}^{2}+\sigma_{\delta}^{2} D_{x}^{2}}, \quad Y=\sigma_{y},
$$

and

$$
\sigma_{\delta}=\sqrt{\left(\frac{\sigma_{z}^{\prime}}{\eta}\right)^{2}+\left(\frac{\varepsilon_{z}}{\eta \sigma_{z}}\right)^{2}}, \quad \sigma_{z}=\sqrt{5} z_{m} .
$$

Here, $K_{\mathrm{sc}}$ is the transverse space charge perveance, defined by $K_{\mathrm{sc}}=2 N_{L} Z^{2} r_{p} /\left(A \beta^{2} \gamma^{3}\right)$, with $N_{L}$ the number of particles per unit length (line density). $\kappa_{x 0, y 0}$ and $\kappa_{z 0}$ are the linearized external focusing gradients in transverse and longitudinal plane, respectively. $\sigma_{x, y}$ denote the transverse rms betatron beam size; $X$ and $Y$ the transverse rms total beam size with dispersion; $\sigma_{\delta}$ the rms momentum spread; $\sigma_{z}$ is the half of the rms bunch length; $\varepsilon_{d x, d y}$ the generalized transverse rms emittances [21], and $\varepsilon_{z}$ the longitudinal rms emittance defined in Eq. (3).

\section{PIC simulations}

A major consideration of PIC-simulation of bunch compression is the treatment of space charge. For typical 
parameters of bunch compression in synchrotrons, as discussed above, since the bunch length after bunch compression is as many times larger than the transverse beam size, the treatment of space charge in the simulation can be split into a 1D longitudinal calculation and a 2D transverse calculation, which is often referred to as " $2.5 \mathrm{D}$ " in literature (see, for example in Ref. [22]). In the 2.5D PICsimulation, the number of grid points for 2D transverse space charge is chosen to be sufficiently large to reduce the grid dependent noise [23], and the number of slices for the 1D longitudinal space charge are checked by obtaining a smooth longitudinal space charge force. Another consideration is the generation of the initial particle distribution. The longitudinal one can be chosen as parabolic, which is - at least initially - a self-consistent model. In order to compare with the envelope approach, the external longitudinal rf focusing force is chosen to be linear. In the transverse plane, the initial distribution is chosen as a Gaussian (truncated at $3 \sigma$, here $\sigma$ is the standard deviation).

For bunches different longitudinal positions experience different transverse space charge forces, and thus different space-charge modified lattice functions. In the simulation performed here, we adopt the approximation that the initial bunch is transversely rms-matched with lattice functions modified by space charge at the longitudinal bunch center. The beam current at the center of a parabolic bunch takes the form $I=\rho_{L 0} \beta_{0} c=3 N Z q \beta_{0} c /\left(4 z_{m}\right)$. Here $N$ is the number of particles in the bunch and $\rho_{L 0}=3 \mathrm{Nq} /\left(4 z_{m}\right)$ the linear charge density at the bunch center. Substituting the beam current into Eqs. (11)-(14), the matched rms beam size can be calculated numerically.

\section{BUNCH COMPRESSION IN THE SIS-18}

The lattice of the SIS-18 consists of 12 periodic cells formed by the sequence $\{\mathrm{QFB} Q \mathrm{QDB}\}$, in which $\mathrm{QF}$ and QD denote focusing and defocussing quadrupoles respectively, and B represents the dipoles. The assumed type of ion is $U^{73+}$. The related parameters for bunch compression are listed in Table I (See, Ref. [2]). The dependent quantities are shown within brackets in Table I. In our example here, in order to show the space charge limitation, the number of particles per bunch is set to $1 \times 10^{11}$, which is 5 times larger than the typical operational value $\left(2 \times 10^{10}\right)$. Note that we are using an idealized lattice for the SIS-18 ignoring magnet multipole and errors. Including these errors of possible beam degradation for bunch compression requires additional studies.

\section{A. Simulation results}

The simulation results of the particle distributions at a fixed potion (at the entrance of QF) are recorded. Figures 2 and 3 show the particle distributions before (the first turn) and after (the 77th turn) bunch compression. It can be seen that the bunch performs a $90^{\circ}$ rotation during bunch
TABLE I. Main parameters for an example bunch compression in SIS-18.

\begin{tabular}{lcc}
\hline \hline Parameters [unit] & Symbol & Value \\
\hline Atomic mass & $A$ & 238 \\
Charge state & $Z$ & 73 \\
Kinetic energy [MeV/u] & $E_{k}$ & 295 \\
Number of particles per bunch & $\mathrm{N}$ & $1.0 \times 10^{11}$ \\
Circumference [m] & $\mathrm{L}$ & 216 \\
Slip factor & $\eta$ & -0.5 \\
Bare tune & $Q_{0, x, y}$ & $4.27,3.47$ \\
Harmonic number & $h$ & 1 \\
Initial half bunch length [m] & $z_{m, i}$ & 78 \\
(Initial half bunch length [ns]) & $\tau_{i}$ & 395 \\
Initial rms momentum spread & $\sigma_{\delta, i}$ & $5 \times 10^{-4}$ \\
Final half bunch length [m] & $z_{m, f}$ & 6.0 \\
(Final half bunch length [ns]) & $\tau_{f}$ & 30.0 \\
(Ideal final rms momentum spread) & $\sigma_{m, f}$ & $6.4 \times 10^{-3}$ \\
(rf voltage [kV]) & $V_{\mathrm{rf}}$ & 68 \\
(Required turns) & $n$ & 77 \\
Transverse rms emittances [mm mrad] & $\varepsilon_{x, y}$ & 15,5 \\
Phase advance of one periodic cell [deg] & $k_{0 x, 0 y}$ & 128,104 \\
\hline \hline
\end{tabular}

compression. After compression, compared with Fig. 2, the vertical emittance and vertical beam size in Fig. 3 stay almost constant; the horizontal emittance growth and enlarged horizontal beam size is from the increasing momentum spread and dispersion during bunch compression and is proven to be reversible, as discussed later in Fig. 6. Here and in the following text the transverse emittances are understood as rms emittances.
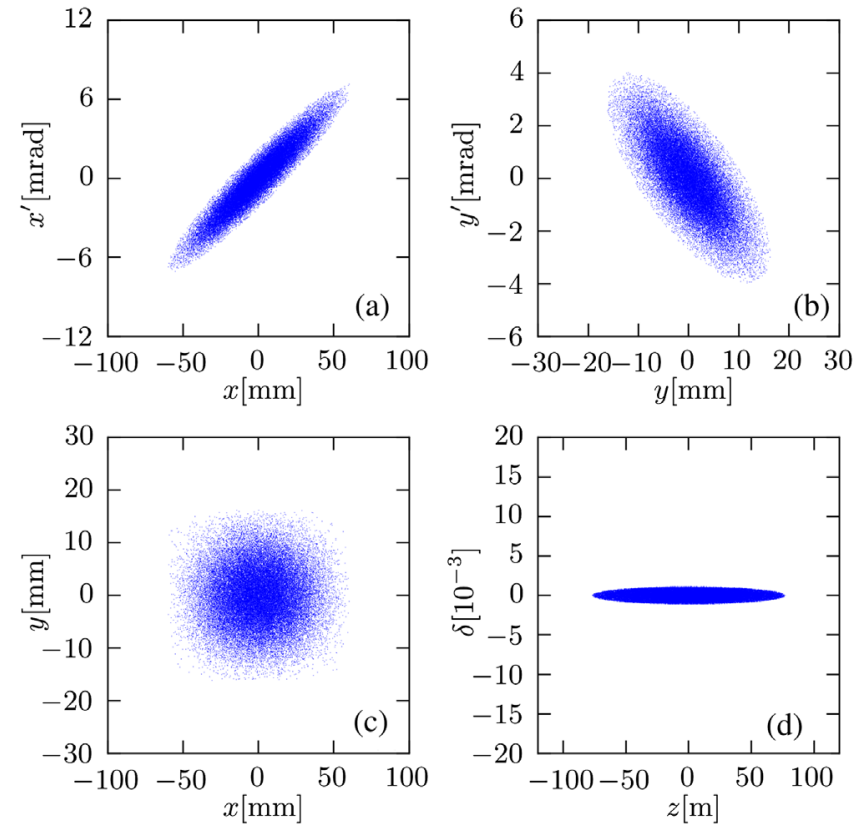

FIG. 2. Particle distribution in (a) $x-x^{\prime}$ phase space; (b) $y-y^{\prime}$ phase space; (c) $x-y$ space and (d) longitudinal $z-\delta$ phase space before bunch compression. 

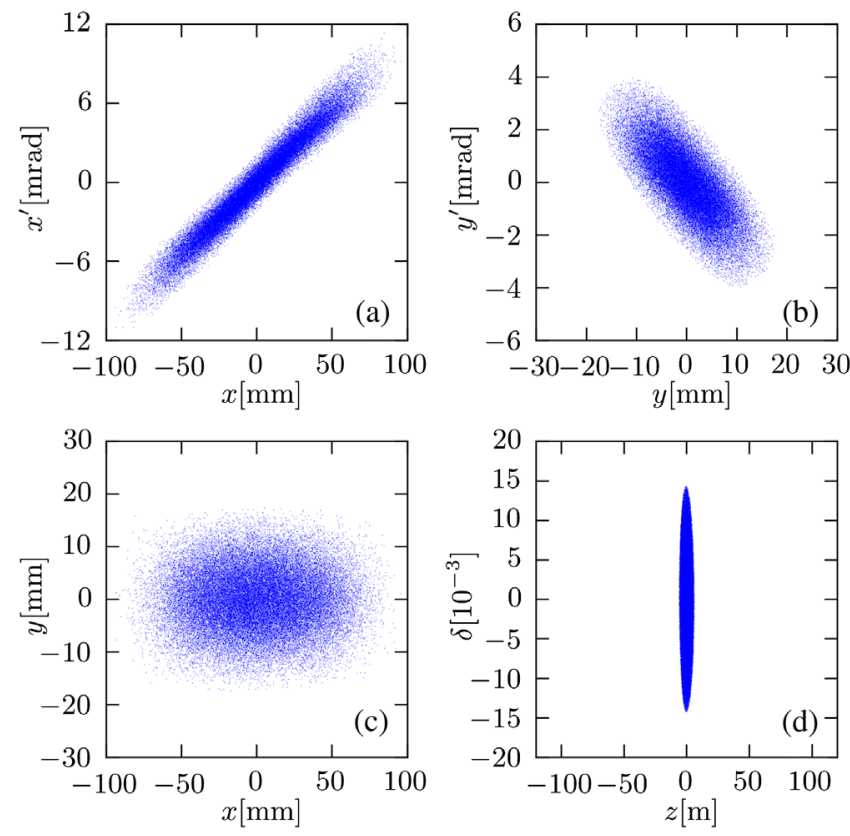

FIG. 3. Particle distribution in (a) $x-x^{\prime}$ phase space; (b) $y-y^{\prime}$ phase space; (c) $x-y$ space; and (d) longitudinal $z-\delta$ phase space after bunch compression.

\section{B. Envelope solutions}

With the initial parameters listed in Table I, the coupled longitudinal-transverse envelope equation set in Eqs. (11)-(14) can be solved numerically. As discussed above, since the synchrotron motion is much slower than the betatron motion during bunch compression, the transverse beam size $X$ can be kept matched to the space-charge modified lattice and dispersion for every turn. In practice, the numerical solution is found as follows: first, the half bunch length $z_{m}$ for each turn is calculated by the longitudinal envelope equation, from which one can obtain by Eq. (16) the beam current and momentum spread for each turn during bunch compression. Second, based on the beam current, the transverse space charge perveance at each turn $K_{\mathrm{sc}}(n)$ is calculated. Using the transverse envelope equation set in Eqs. (11)-(14) and Eq. (15), the evolution of the matched total rms beam sizes $X(n), Y(n)$, the betatron beam sizes $\sigma_{x}(n), \sigma_{y}(n)$ and the space-charge-modified dispersion $D_{x}(n)$ and its derivative $D_{x}^{\prime}(n)$ (at the fixed position: the entrance of $\mathrm{QF}$ ) during compression are obtained, which are all functions of number of turns.

\section{Comparison of simulation and envelopes}

The numerical solutions of bunch length $z_{m}$ are shown in Fig. 4 and compared with the simulation results. It can be seen that the evolution of bunch length during bunch compression from the envelope approach is in almost perfect agreement with the simulation results.

Figure 5 shows the transverse results from the two approaches. The blue solid line and blue dotted line are

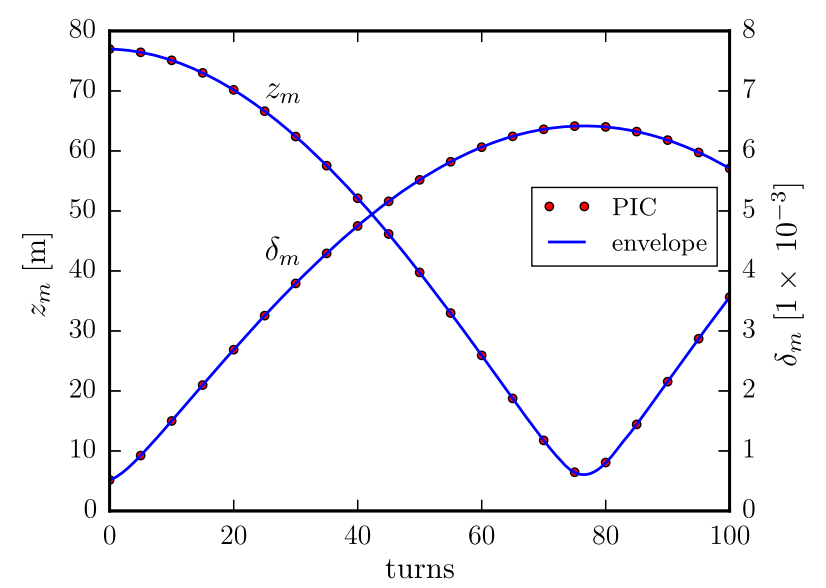

FIG. 4. Evolution of bunch length $z_{m}$ and rms momentum spread $\delta_{m}$ during bunch compression obtained from PIC simulation and envelope approach.

the transverse rms beam size $(X)$ and betatron rms beam size $\left(\sigma_{x}\right)$ solved from the coupled envelope method. The red solid line and red dotted line are the transverse rms beam size and betatron rms beam size obtained from the simulation. The former can be obtained directly by the definition of the rms quantity: $X=\frac{1}{N}\left(\sum_{i=1}^{N} x_{i}^{2}\right)^{1 / 2}$, while the latter is obtained by $\sigma_{x}=\frac{1}{N}\left[\sum_{i=1}^{N}\left(x_{i}-\delta_{i} D_{x}\right)^{2}\right]^{1 / 2}$. Here $x_{i}$ denotes the horizontal offset of the $i^{\text {th }}$ macroparticle, $N$ the total number of macroparticles in the simulation and $D_{x}$ the space-charge-modified dispersion solved from the envelope approach. It can be seen from Fig. 5 that during bunch compression the total horizontal beam size $X$ is increasing due to the increasing dispersion, while the betatron beam size $\sigma_{x}$ remains constant. The results of transverse beam sizes from the envelope approach agree well with the results from PIC simulations. The spacecharge-modified dispersion can also be evaluated from PIC

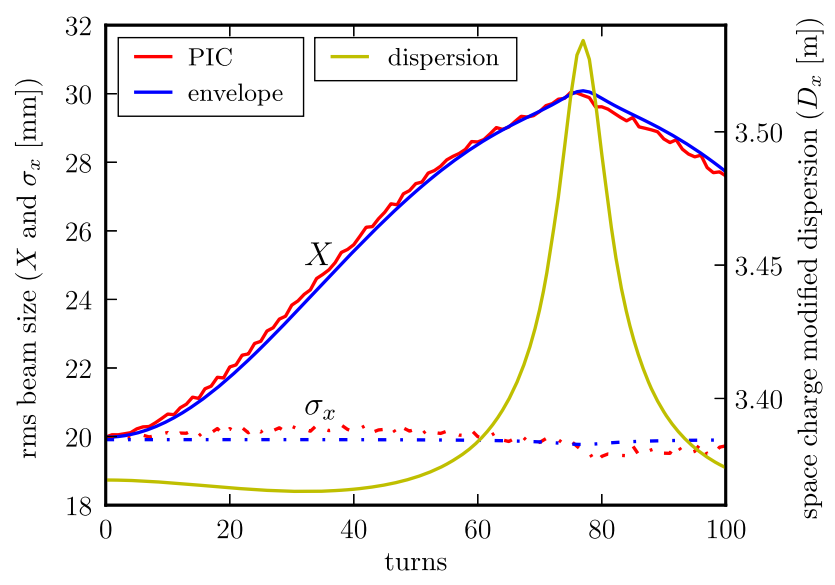

FIG. 5. Evolution of the total $\mathrm{rms}$ beam size $X$, the betatron beam size $\sigma_{x}$, and the space-charge-modified dispersion $D_{x}$ during bunch compression obtained from PIC simulation and envelope approach. 
simulations by $D_{x}=\sum_{i=1}^{N}\left(x_{i} \delta_{i}\right) /\left(\sum_{i=1}^{N} \delta_{i}^{2}\right)$. In our simulations the evolution of the space-charge-modified dispersion (not shown here) obtained from the above equation agrees with the one obtained from the envelope approach at the second half of the compression process (after 45 turns), but large noisy oscillations are observed at the first half of the process, which need to be further studied and improved.

The dispersive increase in $\epsilon_{d x}$ (Fig. 3) and in $X$ (Fig. 5) are, in principle, reversible and expected to vanish in a dispersion-free section. To illustrate this point, we consider a $180^{\circ}$ rotation, with an initial $90^{\circ}$ compression followed by a $90^{\circ}$ decompression. As plotted in Fig. 6, the horizontal emittance $\epsilon_{x}$ is obtained directly from simulations, while the generalized horizontal emittance $\epsilon_{d x}$ is calculated based on the coordinate transformation with $x_{i}-\delta_{i} D_{x}, x_{i}^{\prime}-\delta_{i} D_{x}^{\prime}$ [21,24], in which $D_{x}$ and $D_{x}^{\prime}$ are obtained from the coupled envelope approach. It can be observed that both the horizontal emittance $\epsilon_{x}$ and the generalized one, $\epsilon_{d x}$, are fully reversible after a $180^{\circ}$ rotation. Compared to the large dispersive growth of the horizontal emittance $\epsilon_{d x}$ stays almost constant during bunch compression. Only a slight growth of $\epsilon_{d x}$ is observed at the end of the compression (see the arrowhead in Fig. 6), where space charge reaches its maximum value. We attribute this to the fact that the spacecharge modified dispersive $D_{x}$ and its derivative $D_{x}^{\prime}$ are obtained from the coupled envelope approach with a linear space charge, and assumed to be constant for all particles. For the Gaussian beam in the simulation, however, the space charge is nonlinear and different particles have different values of space-charge modified dispersion. It can be assumed that if the space-charge modified dispersion and its derivative $D_{x}^{(i)}, D_{x}^{\prime(i)}$ are exactly known for each particle, the generalized emittance $\epsilon_{d x}$ is ideally an invariant after the coordinate transformation $x_{i}-\delta_{i} D_{x}^{(i)}$, $x_{i}^{\prime}-\delta_{i} D_{x}^{\prime(i)}$. In other words, the fact that we use the spacecharge modified dispersion from an envelope (KV) model to evaluate the generalized emittance with nonlinear space charge leads to this residual dispersive growth of the

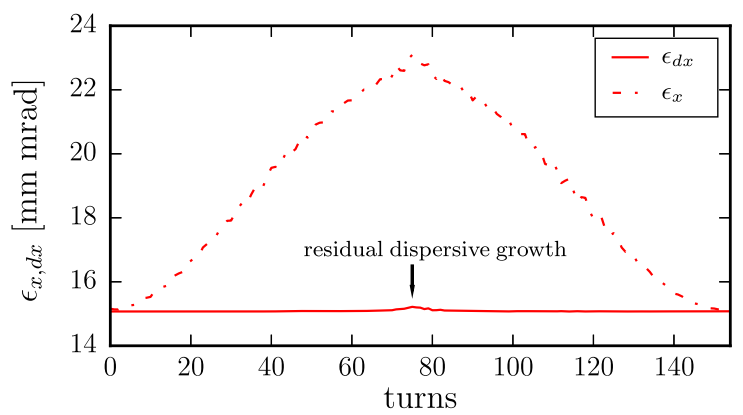

FIG. 6. The evolution of horizontal emittance $\epsilon_{x}$ and generalized horizontal emittance $\epsilon_{d x}$ during a $180^{\circ}$ rotation in the longitudinal phase space. generalized horizontal emittance, which is usually small and reversible.

\section{ENHANCED SPACE CHARGE EFFECTS DURING BUNCH COMPRESSION IN SIS-18}

The PIC simulation and numerical results in Figs. 3 and 5 respectively illustrate that with the parameters listed in Table I only dispersive emittance growth is observed, and the betatron beam size $\sigma_{x}$ remains almost constant during the bunch compression in SIS-18.

With higher intensity $-k_{y}$ getting close to $90^{\circ}$ and beyond the case of Table I- can lead to a fourth order single particle resonance driven by space charge, possibly also the well-known envelope instability. In the horizontal plane the recently discovered dispersion-induced instability could be induced when $k_{x}$ is approaching and crossing $120^{\circ}$. In this section we have doubled the bunch intensity in Sec. IVA and reduced the horizontal phase advance in Sec. IV B in order to investigate these issues and their possible impact on space charge limitations during bunch compression in the SIS-18.

\section{A. $90^{\circ}$-related intensity limitation}

In the stop band related to a transverse $90^{\circ}$ phase advance per focusing period the simultaneous appearance of an envelope instability (see, for example, Ref. [25]) with a fourth order single particle space charge resonance has more recently been studied in straight lattices and for short bunches [20,26-28].

In order to investigate the $90^{\circ}$-related intensity limitation during compression of long bunches, we double bunch intensity (from $N=1 \times 10^{11}$ to $2 \times 10^{11}$ ) in Table I and keep other parameters unchanged. By doing this, the vertical depressed phase advance $k_{y}$ will cross the $90^{\circ}$ line during bunch compression.

Figure 7 shows as solid line the result of depressed phase advances by solving the coupled envelope equation of Eqs. (11)-(14) with doubled bunch intensity. As can be seen, during bunch compression $k_{x}$ is always above $120^{\circ}$, while $k_{y}$ crosses the $90^{\circ}$ line after turn 71. Particle distributions at turn 71 from PIC simulation are plotted in Fig. 8. One can see a clear fourfold structure in $y-p_{y}$ phase space, characterizing the fourth-order resonance, with the condition

$$
4 k_{y}=360^{\circ} \text {, }
$$

which is consistent with the numerical results in Fig. 7. Particle distributions in $y-p_{y}$ phase space at three representative turns (68th, 72nd, 76th) are shown in Fig. 9. The formation of four islands starts at turn 68. With space charge increasing, they move away from the particle core, leading to an increasing emittance. The four islands in Figs. 8 and 9 confirm that the emittance growth during 


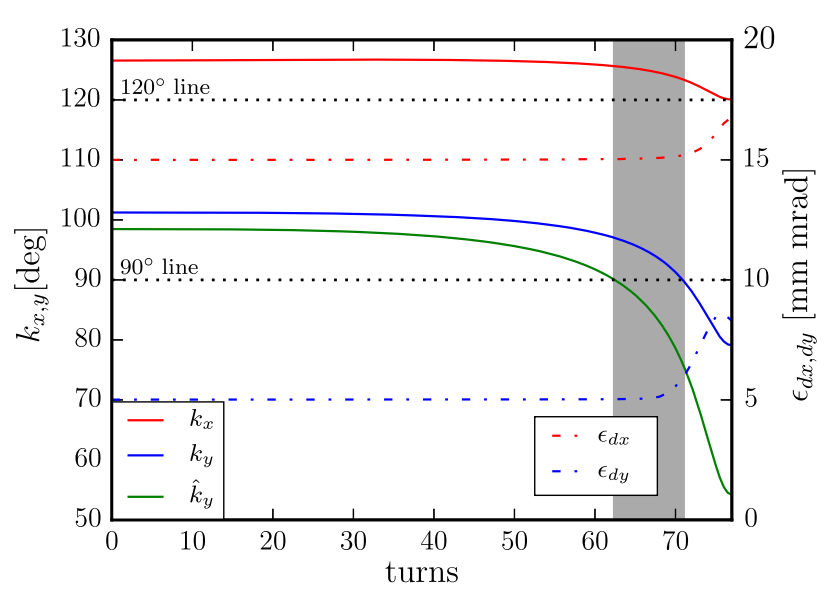

FIG. 7. Space-charge depressed phase advances $k_{x, y}$ (solid lines) solved from the coupled envelope approach and emittance growth $\epsilon_{d x, d y}$ (dashed lines) from PIC simulations during bunch compression with doubled bunch intensity. (Shaded area denotes the range from $k_{y}=90^{\circ}$ to $\hat{k}_{y}=90^{\circ}$.)

bunch compression is due to the fourth-order resonance, and no envelope instability is observed in PIC simulations.

The evolution of transverse emittances in simulation during bunch compression is shown as dashed line in Fig. 7. Note that the emittances start to increase at turn 68, slightly before turn 71 , where $k_{y}=90^{\circ}$. Also note that for a Gaussian beam $k_{y}$ is understood as the depressed tune of an equivalent KV beam. In fact, for a Gaussian beam, particles have spread out depressed phase advances, and most
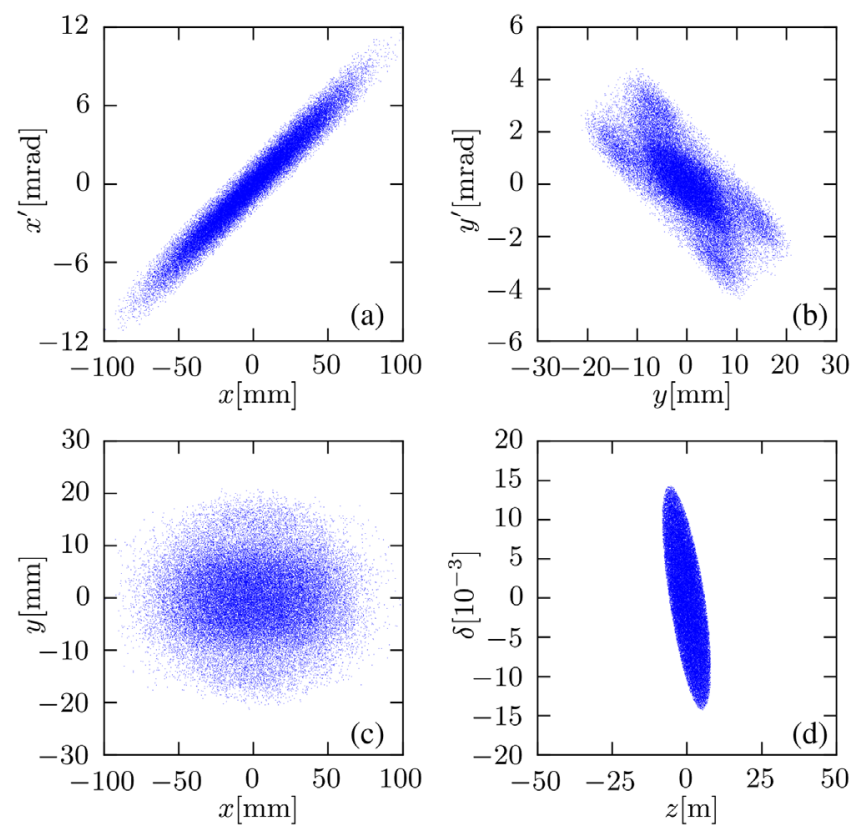

FIG. 8. Particle distribution in (a) $x-x^{\prime}$ phase space; (b) $y-y^{\prime}$ phase space; (c) $x-y$ space; and (d) longitudinal $z-\delta$ phase space at turn 72 during bunch compression, with doubled bunch intensity.

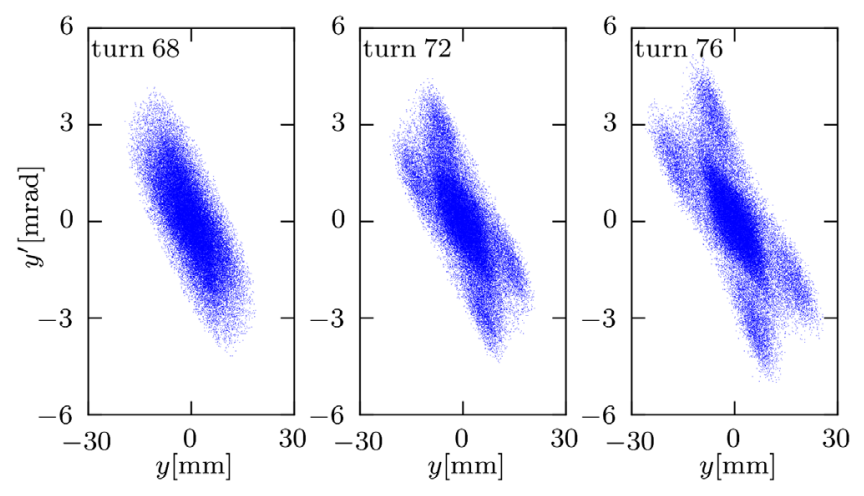

FIG. 9. The evolution of particle distribution in the $y-y^{\prime}$ phase space at final stage of bunch compression with double bunch intensity at 68th, 72nd, 76th turns during bunch compression.

particles are located in the range from $k_{y}$ to $\hat{k}_{y}$, (here $\hat{k}_{y}=k_{0, y}-2 \Delta k_{y}$ denotes the maximum depressed phase advance in a Gaussian beam) as shown schematically in Fig. 10. Therefore, an individual particle (marked as $i$ th, with its phase advances $k_{y}^{i}$ ) will arrive at the $90^{\circ}$ line before turn 71 when satisfying

$$
4 k_{y}^{i}=360^{\circ} \quad\left(k_{y} \geq k_{y}^{i} \geq \hat{k}_{y}\right) .
$$

This leads to the fourth-order resonance of the $i$ th particle and contributes to the initial emittance growth.

In order to further study the mechanism of the $90^{\circ}$-related intensity limitation, and distinguish the role of fourth-order resonance and envelope instability during bunch compression, we calculate the envelope modes (the fast mode $\phi_{1}$, the slow mode $\phi_{2}$ ) and dispersion mode $\phi_{d}$ [16] during bunch compression from the coupled envelope model, as shown in Fig. 11. With space charge increasing, $\phi_{2}$ will drop and lock on the $180^{\circ}$ line after turn 72 when $k_{y}<90^{\circ}$, indicating the occurrence of envelope instability. However, simulation results in Fig. 9 show that from turn

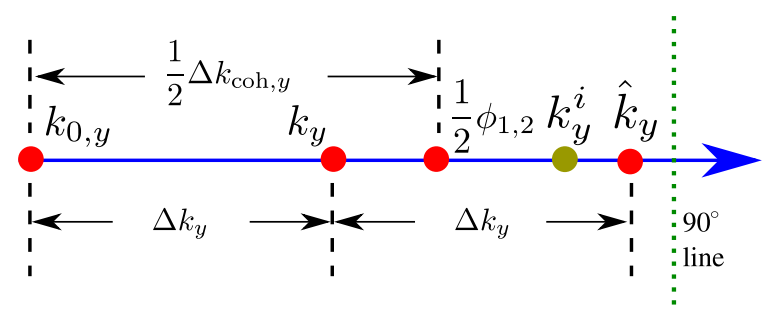

FIG. 10. Schematic drawing of the comparison on location of the phase advances to the phase shifts of envelope modes $\phi_{1,2}$. $k_{0, y}$ is the phase advance without space charge; $k_{y}$ is the depressed phase advance shifted by $\Delta k_{y}$ for an equivalent $\mathrm{KV}$ beam; $\hat{k}_{y}$ is the most depressed space charge shifted by $2 \Delta k_{y}$ in a Gaussian beam; $\Delta k_{\text {coh }, y}$ is the coherent space charge tune shift; $k_{y}^{i}$ is the phase advance of an individual particle. The arrow indicates the direction of increasing space charge. Dashed line denotes the $90^{\circ}$ phase advance line (in Fig. 7) during bunch compression. 


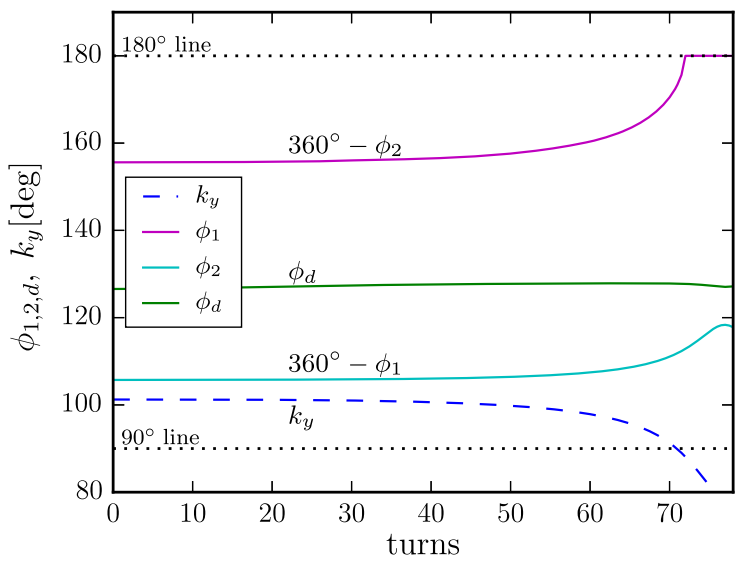

FIG. 11. Phase shifts of the envelope modes $\phi_{1,2}$ and the dispersion mode $\phi_{d}$ numerically solved from the coupled envelope approach during bunch compression.

72 to turn 76 only the fourth-order resonance is observed. It is pointed out in Refs. [26,28] that for a well-matched Gaussian beam transported for a short distance the fourthorder resonance can dominate over the envelope instability, since the fourth-order resonance readily occurs from the beginning due to the presence of the "pseudo-octupole" in the initial density profile. In contrast, for the envelope instability, it takes more time to "amplify" the initial noise and develop the collective instability.

To illustrate this, we plot schematically the relative positions the phase advances with and without space charge, the phase shifts of envelope modes, and the coherent space charge tune shift $\Delta k_{\text {coh, } y}$ obtained from envelope approach. The envelope instability in Fig. 11 can be described as

$$
\phi_{2}=2 k_{0, y}-\Delta k_{\mathrm{coh}, y}=\frac{1}{2} 360^{\circ} .
$$

During bunch compression, with space charge increasing, all quantities except $k_{0, y}$ in Fig. 10 will decrease and dynamically move towards the $90^{\circ}$ line. Clearly, the most depressed phase advance $\hat{k}_{y}$ will firstly arrive at the $90^{\circ}$ line. Then particles with $k_{y}^{i}$ in the region of $\frac{1}{2} \phi_{2}<k_{y}^{i}<\hat{k}_{y}$ can trigger the fourth-order resonance before $\frac{1}{2} \phi_{2}$ arrives at the $90^{\circ}$ line. The resulting emittance growth can weaken the space charge effect and prevent $\frac{1}{2} \phi_{2}$ to approach the $90^{\circ}$ line. In other words, individual particles can firstly arrive at the $90^{\circ}$ resonance line, leading to the fourth-order resonance and prevent inducing the envelope instability.

In order to avoid the onset of the fourth-order resonance under the intensity of $N=2 \times 10^{11}$, we adjust the vertical phase advance from $k_{0, y}=104^{\circ}$ to $118^{\circ}$. In Fig. 12 we show the emittance growths from simulation and spacecharge modified phase advances from the coupled envelope equations. The phase advance $k_{y}$ solved from the envelope approach stays above $90^{\circ}$, and the vertical emittance remains almost constant. From turn 74, the slight emittance growth is from those particles with $\hat{k}_{y}<90^{\circ}$. It is worth pointing out that in both, Figs. 7 and 12, a slight increase of $\epsilon_{d x}(10 \%)$ can be observed. We attribute this to the same reason as observed in Fig. 6: the residual dispersive emittance growth. Clearly, with doubled bunch intensity, the residual dispersive emittance growth $(13 \%)$ is much larger compared to the one (1\%) in Fig. 6 because of the enhanced space charge effects.

\section{B. $120^{\circ}$-related intensity limitation}

During bunch compression space charge as well as dispersion are increasing. With sufficiently high bunch intensity and designed horizontal phase advance $k_{0, x}$ larger than $120^{\circ}$, as is the case for SIS-18, the combined effects of space charge and dispersion could induce the $120^{\circ}$ dispersion instability, which is another limitation factor for bunch compression. In order to investigate this, we adjust $k_{0, x}$ from $128^{\circ}$ to $122^{\circ}$ in Table I and keep other parameters unchanged. By doing this, the horizontal phase advance $k_{x}$ will cross the $120^{\circ}$ line during bunch compression.

Particle distributions at turn 70 from PIC simulation are plotted in Fig. 13, which is similar to the case in Fig. 3, and no evident emittance growth has occurred up to this point. Figure 14 shows the result of depressed phase advances by solving the coupled envelope equation of Eq. (11) with $k_{0, x}=122^{\circ}, k_{0, y}=104^{\circ}$. As can be seen, during bunch compression $k_{y}$ is always above $90^{\circ}$ while $k_{x}$ crosses the $120^{\circ}$ line after 70 th turn.

Particle distributions in $x-p_{x}$ phase space at three representative turns (72nd, 74th, and 76th) are shown in

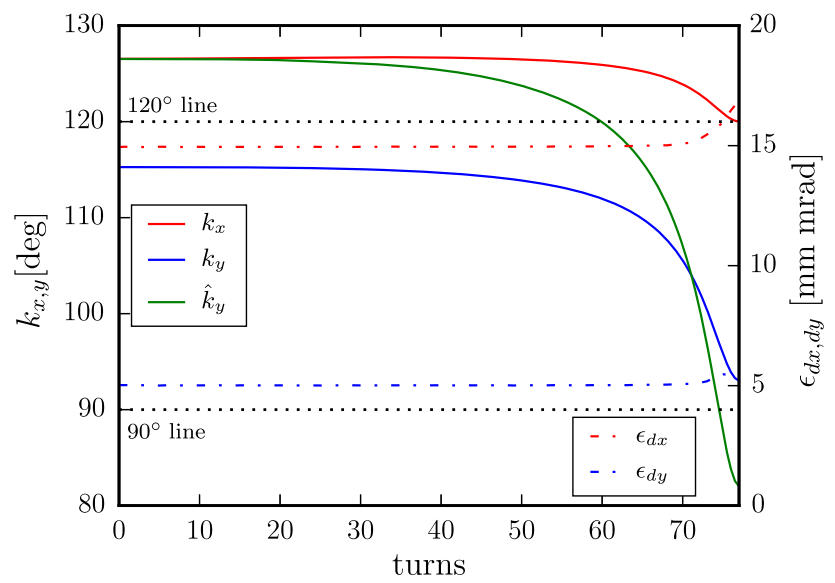

FIG. 12. Space-charge depressed phase advances $k_{x, y}$ (solid lines) solved from the coupled envelope approach and emittance growth $\epsilon_{d x, d y}$ (dashed line) from PIC simulations during bunch compression with doubled bunch intensity and $k_{0, x}=128^{\circ}$, $k_{0, y}=118^{\circ}$. 

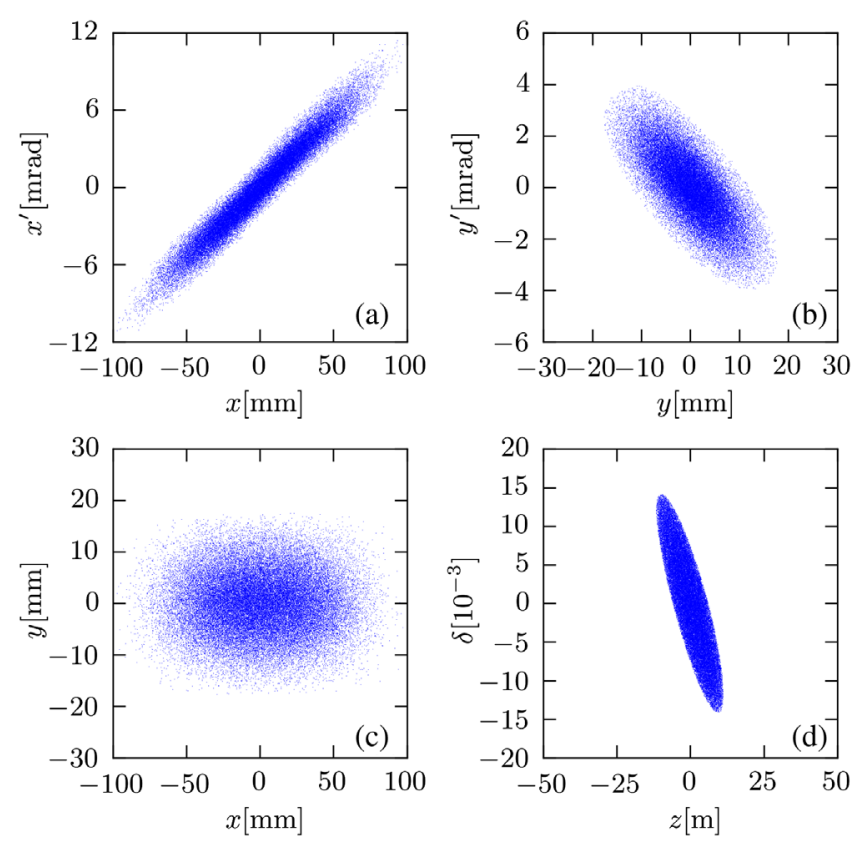

FIG. 13. Particle distribution in (a) $x-x^{\prime}$ phase space; (b) $y-y^{\prime}$ phase space; (c) $x-y$ space; and (d) longitudinal $z-\delta$ phase space at 70th turn during bunch compression, with $k_{0, x}=122^{\circ}, k_{0, y}=104^{\circ}$.

Fig. 15, from which one can see the emittance deformation and the effect of emittance growth.

The evolution of the transverse emittance in simulation during bunch compression is shown as dashed line in Fig. 14. Note that sharp increasing of the emittance occurs starting from turn 72. This is different from the case when crossing the $90^{\circ}$ resonance line in Fig. 7, in which emittance growth occurs much earlier than $k_{y}=90^{\circ}$. We attribute this difference to the reason that the emittance growth in Fig. 13 is from the $120^{\circ}$ dispersion instability, which is a collective effect with

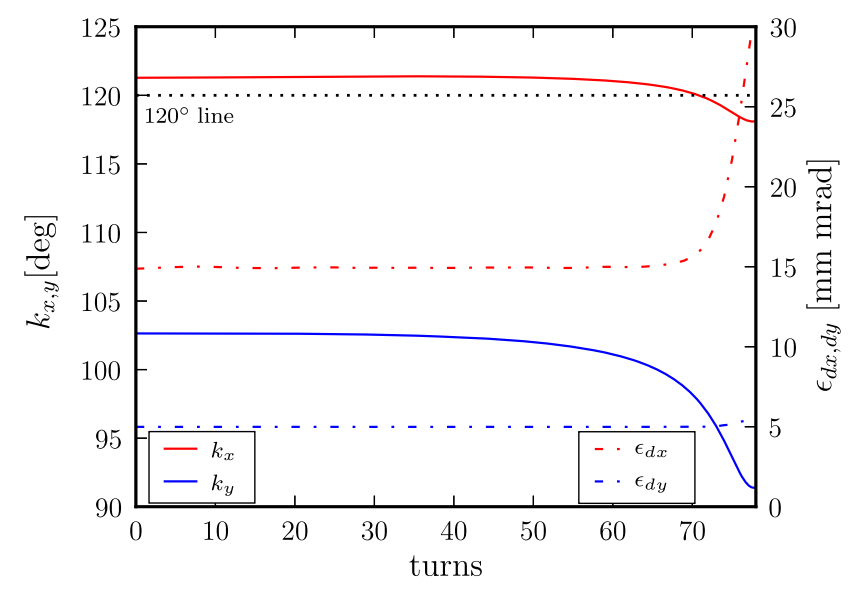

FIG. 14. Space-charge depressed phase advances $k_{x, y}$ (solid lines) obtained from the coupled envelope approach and emittance growth $\epsilon_{d x, d y}$ (dashed lines) from PIC simulations during bunch compression with $k_{0, x}=122^{\circ}$ and $k_{0, y}=104^{\circ}$.

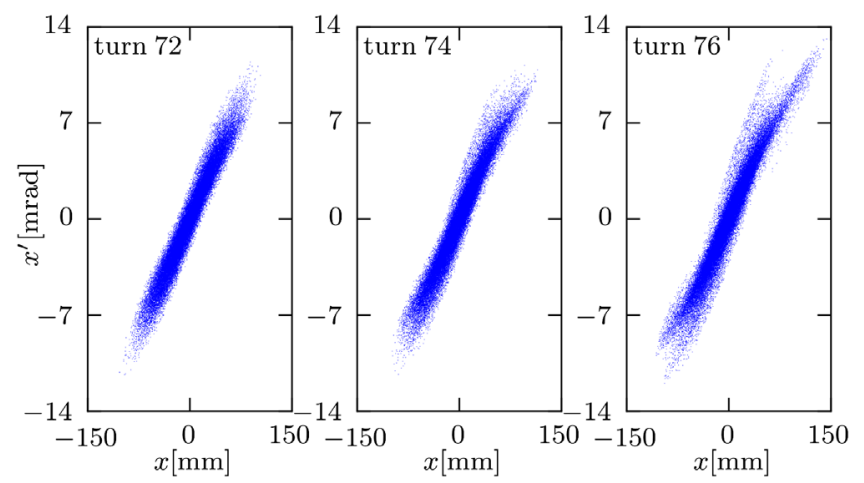

FIG. 15. Evolution of the particle distribution in the $x-x^{\prime}$ phase space at final stage of bunch compression with $k_{0, x}=122^{\circ}$, $k_{0, y}=104^{\circ}$ at 72 th, 74 th, 76 th turns during bunch compression.

$$
\phi_{1}+\phi_{d}=360^{\circ}
$$

when

$$
k_{0, x}>120^{\circ} \text { and } k_{x}<120^{\circ} .
$$

The emittance growth observed in Fig. 14 must be distinguished from the residual dispersive growth of $\epsilon_{d x}$ appeared in Figs. 6, 7, and 12. The point is that, with the same bunch intensity, the horizontal emittance growth $(100 \%)$ in Fig. 14 is much larger than that in Fig. 6 (1\%), Fig. 6 (13\%), and Fig. 7 (13\%), and can be explained only by the dispersion-induced instability. Moreover, the dispersive emittance growth is characterized by an extension of emittance along the slope of the ellipse of the emittance in the $x-x^{\prime}$ plane, as shown in Figs. 3 and 13. In comparison, the evolution of particle distribution in Fig. 15 has no such regular extension and behaves like the emittance growth during the envelope instability (for example, see the figures in [25] and Fig. 2 in [26]).

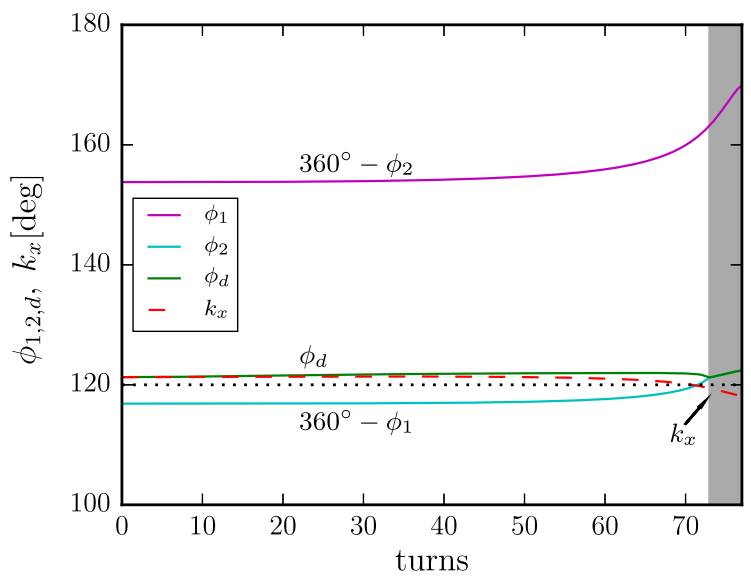

FIG. 16. Phase shifts of the envelope modes $\phi_{1}, \phi_{2}$ and dispersion mode $\phi_{d}$ numerically solved from the coupled envelope approach during bunch compression with $k_{0, x}=122^{\circ}$ and $k_{0, y}=104^{\circ}$. (Shaded area denotes the stop band of the dispersion-induced instability.) 
In order to further study the mechanism of $120^{\circ}$-related intensity limitation, we calculate the fast mode $\phi_{1}$, slow mode $\phi_{2}$ and dispersion mode $\phi_{d}$ with $k_{0, x}=122^{\circ}$, $k_{0, y}=104^{\circ}$. As shown in Fig. 16, with space charge increasing during bunch compression, the curve $\phi_{d}$ will be confluent with $360^{\circ}-\phi_{1}$, indicating the dispersioninstability, which is in good agreement with the sharp emittance growth at turn 71 in Fig. 14.

The onset of the dispersion-induced instability is not inhibited compared with that of the envelope instability. Firstly, compared with the competition between fourthorder resonance and envelope instability related to $90^{\circ}$, there is no obvious single particle resonance, which exists earlier and can weaken the space charge and prevent the $120^{\circ}$ dispersion instability. This is different from the case of the $90^{\circ}$ envelope instability, which is suppressed by the competition with the fourth-order resonance. Second, the bunch intensity threshold of the $120^{\circ}$ dispersion instability is much lower than that of the $90^{\circ}$ envelope instability, as can be seen in Fig. 2 in Ref. [16]. Therefore, the former instability would need less time to develop than the latter.

\section{CONCLUSIONS AND OUTLOOK}

The coupled longitudinal-transverse envelope equations including dispersion are applied to the bunch compression in SIS-18. The analytical results are found to agree well with PIC simulation results. Results show two major intensity limitations caused by space charge during bunch compression when phase advances dynamically cross $90^{\circ}$ or $120^{\circ}$. When crossing $90^{\circ}$, the fourth-order resonance dominates over the envelope instability and leads to emittance growth. When crossing $120^{\circ}$, simulation results show that the recently discovered dispersion-induced instability could be another intensity limitation for bunch compression. The agreement between envelope solutions and simulation results shows that the coupled longitudinal-transverse envelope approach defines the stop band of the $120^{\circ}$ dispersion instability, which should be avoided during bunch compression. Future bunch compression studies will have to include also other potential intensity limiting mechanisms, like for example machine errors and impedances.

\section{ACKNOWLEDGMENTS}

One of the authors (Y. S. Yuan) would like to thank HGS-HIRe for FAIR at GSI for financial support for doctoral training.

[1] http://www.fair-center.eu/.

[2] O. Chorniy, O. Boine-Frankenheim, P. Hülsmann, and P. J. Spiller, Fast compression of intense heavy-ion bunches in SIS-18, in Proceedings of HB2010, Morschach, Switzerland, 2010, p. 686, https://accelconf.web.cern.ch/ accelconf/HB2010/papers/tho1d03.pdf.
[3] P. Hülsmann, R. Balss, U. Laier, and H. Klingbeil, Bunch compression for FAIR, in Proceedings of 2009 Particle Accelerator Conference, Vancouver, Canada, 2009, p. 864, https://accelconf.web.cern.ch/accelconf/ pac2009/papers/tu5pfp023.pdf.

[4] O. Boine-Frankenheim, Accelerator challenges of proposed radioactive beam facilities, in Proceedings of the 8th European Particle Accelerator Conference, Paris, 2002 (EPS-IGA and CERN, Geneva, 2002), p. 99, http://accelconf.web.cern.ch/AccelConf/e02/PAPERS/ FRXGB001.pdf.

[5] K. Blasche, O. Boine-Frankenheim et al., Bunch compression in the heavy ion synchrotron at GSI, Bunch compression in the heavy ion synchrotron SIS at GSI, in Proceedings of the 6th European Particle Accelerator Conference, Stockholm, 1998 (IOP, London, 1998), p. 1347, http://accelconf.web.cern.ch/AccelConf/e98/ PAPERS/THP41G.PDF.

[6] S. M. Lund, O. Boine-Frankenheim, G. Franchetti, I. Hofmann, and P. Spiller, Simulations of axial bunch compression in heavy ion rings for plasma physics applications at GSI, in Proceedings of the 1999 Particle Accelerator Conference, New York, 1999 (IEEE, New York, 1999), p. 1785.

[7] K. Y. Ng, Space-charge effects on bunch rotation in the longitudinal phase space, Proceedings of the 2001 Particle Accelerator Conference, Chicago, Illinois, USA, 2001 (IEEE, Piscataway, NJ, 2001) p. 2893, http://accelconf .web.cern.ch/AccelConf/p01/PAPERS/RPAH022.PDF.

[8] T. Kikuchi, M. Nakajima, K. Horioka, and T. Katayama, Beam instability induced by space charge oscillation during final beam bunching for heavy ion inertial fusion, Phys. Rev. ST Accel. Beams 7, 034201 (2004).

[9] T. Kikuchi, S. M. Lund, T. Katayama, and S. Kawata, Bunch compression in a ring for future RIKEN projects, Nucl. Instrum. Methods Phys. Res., Sect. A 544, 393 (2005).

[10] T. Kikuchi and K. Horioka, Beam behavior under a nonstationary state in high-current heavy ion beams, Nucl. Instrum. Methods Phys. Res., Sect. A 606, 31 (2009).

[11] K. Y. Ng, Space-charge effects on beam rotation at the compressor ring, Fermilab Report No. FN-0955-APC, 2013 (unpublished).

[12] F. J. Sacherer, RMS Envelope equations with space charge, IEEE Trans. Nucl. Sci. 18, 1105 (1971).

[13] G. Franchetti, I. Hofmann, and G. Rumolo, Effect of space charge on bunch compression near the transition, Phys. Rev. ST Accel. Beams 3, 084201 (2000).

[14] Y. Zou, J. Tang, J. Chen, X. Li, and H. Sun, Short-bunch extraction in a rapid cycling synchrotron, Phys. Rev. ST Accel. Beams 17, 060101 (2014).

[15] I. Hofmann, G. Franchetti, and A. Fedotov, Space charge resonances and instabilities in rings, in Proceedings of the 20th ICFA Advanced Beam Dynamics Workshop on High Intensity and High Brightness Hadron Beams, Batavia, Illinois, AIP Conf. Proc. No. 642 (AIP, New York, 2002), p. 248.

[16] Y. S. Yuan, O. Boine-Frankenheim, G. Franchetti, and I. Hofmann, Dispersion-Induced Beam Instability in Circular Accelerators, Phys. Rev. Lett. 118, 154801 (2017). 
[17] D. Neuffer, Longitudinal motion in high current ion beams-a self-consistent phase space distribution with an envelope equation, IEEE Trans. Nucl. Sci. 26, 3031 (1979).

[18] S. Y. Lee, Accelerator Physics, 3rd ed. (World Scientific, Singapore, 2011).

[19] S. Cousineau, S. Y. Lee, J. A. Holmes, V. Danilov, and A. Fedotov, Space charge induced resonance excitation in high intensity rings, Phys. Rev. ST Accel. Beams 6, 034205 (2003).

[20] J. Qiang, Three-dimensional envelope instability in periodic focusing channels, Phys. Rev. Accel. Beams 21, 034201 (2018).

[21] M. Venturini and M. Reiser, rms Envelope Equations in the Presence of Space Charge and Dispersion, Phys. Rev. Lett. 81, 96 (1998).

[22] A. Shishlo, S. Cousineau, J. Holmes, and T. Gorlov, The particle accelerator simulation code PyORBIT, Procedia Comput. Sci. 51 (2015) 1272.
[23] I. Hofmann and O. Boine-Frankenheim, Grid dependent noise and entropy growth in anisotropic $3 \mathrm{~d}$ particle-in-cell simulation of high intensity beams, Phys. Rev. ST Accel. Beams 17, 124201 (2014).

[24] S. Y. Lee and H. Okamoto, Space-Charge Dominated Beams in Synchrotrons, Phys. Rev. Lett. 80, 5133 (1998).

[25] J. Struckmeier and M. Reiser, Theoretical studies of envelope oscillations and instabilities of mismatched intense charged-particle beams in periodic focusing channels, Part. Accel. 14, 227 (1984).

[26] I. Hofmann and O. Boine-Frankenheim, Space-Charge Structural Instabilities and Resonances in High-Intensity Beams, Phys. Rev. Lett. 115, 204802 (2015).

[27] D. Jeon, J. H. Jang, and H. Jin, Interplay of space-charge fourth order resonance and envelope instability, Nucl. Instrum. Methods Phys. Res., Sect. A 832, 43 (2016).

[28] I. Hofmann and O. Boine-Frankenheim, Parametric instabilities in 3D periodically focused beams with space charge, Phys. Rev. Accel. Beams 20, 014202 (2017). 